\title{
Parametric Macromodels for Efficient Design of Carbon Nanotube Interconnects
}

\author{
Francesco Ferranti, Member, IEEE, Giulio Antonini, Senior Member, IEEE, Tom Dhaene, Senior Member, IEEE, \\ Luc Knockaert, Senior Member, IEEE
}

\begin{abstract}
The continuous increase of the operating frequency and density of integrated circuits leads to consider single wall carbon nanotubes (SWCNTs) and multiwall carbon nanotubes (MWCNTs) as the most promising candidates for future interconnect technology because of their high current-carrying capacity and conductivity in the nanoscale, and immunity to electromigration. Several modeling methods for SWCNT and MWCNT interconnects has been based on the multiconductor transmission line (MTL) theory. These methods are limited to nanostructures with predefined values of electrical and geometrical parameters. Since process technology continues to scale downward and physical interconnect dimensions become smaller, the impact of design parameters (e.g. layout features) on the system behavior has to be carefully investigated for a successful design by performing of design space exploration, optimization and variability analysis. These design activities require multiple system simulations for different values of design parameters and using MTL-based solvers is not an efficient choice. Parametric macromodels can be used to accurately and efficiently model these parameter effects, avoiding the brute-force use of MTLbased solvers.
\end{abstract}

Index Terms-Single wall carbon nanotubes (SWCNTs), multiwall carbon nanotubes (MWCNTs), nano-interconnects, parametric macromodeling, transmission line modeling, crosstalk, transient analysis.

\section{INTRODUCTION}

The increasing progress of very-large-scale integration (VLSI) technology enabled the continuous improvement of the processing speed of electronic circuits. Considering the progressive miniaturization and scaling of semiconductor devices, the need for longer, thinner, and faster interconnects with lower power consumption has become more pronounced. Copper-based interconnects are facing great challenges to satisfy design specifications (reliability, propagation features, crosstalk, etc.) when physical dimensions are scaled down to the nanoscale range. The inherent limitations in metallic interconnections are seriously threatening the future of traditional VLSI industry.

Over the last years, carbon nanotubes (CNTs) have attracted a lot of attention as the most promising candidates for future

Manuscript received August 2013.

Francesco Ferranti, Tom Dhaene and Luc Knockaert are with the Department of Information Technology (INTEC), at Ghent University iMinds, Gaston Crommenlaan 8 Bus 201, B-9050, Gent, Belgium, email: \{francesco.ferranti, tom.dhaene, luc.knockaert\}@ugent.be. Francesco Ferranti is a Post-Doctoral Research Fellow of the Research Foundation Flanders (FWO-Vlaanderen).

Giulio Antonini is with the UAq EMC Laboratory, Dipartimento di Ingegneria Elettrica e dell'Informazione, Università degli Studi dell'Aquila, Via G. Gronchi 18, 67100, L'Aquila, Italy, e-mail: giulio.antonini@univaq.it.

This work has been funded by FWO-Vlaanderen. interconnect technology due to their outstanding electrical, thermal properties and and large-current carrying capability [1]-[5]. The high dc resistance associated with an isolated single-wall CNT (SWCNT) [1] suggests the use of bundles consisting of numerous parallel connected SWCNTs [5]-[9]. Nevertheless, the limits in the selective growth of metallic SWCNTs with controlled morphology makes the use of multiwall CNTs (MWCNTs), a viable solution for intermediate and long interconnects. Interconnects are fundamental subsystems of complex integrated circuits and require to be properly designed, since from the early design stage they can seriously limit transmission data rates or increase the bit error rate beyond acceptable limits. Accurate and efficient modeling of CNT-based interconnections is fundamental to properly analyze and investigate their performances. SWCNT bundles have also been considered as an alternative to copper to constitute via interconnects [10]. In [11], the electrical behavior of vias based on CNT bundles, either SWCNT or MWCNT, are investigated taking the effects of size, temperature, chirality and frequency into account in a consistent way.

Different modeling techniques for SWCNTs and MWCNTs have been based on multiconductor transmission line (MTL) theory in the frequency-domain [12]-[16]. More advanced transmission line models have been proposed in [17], [18] accounting for intershell tunneling and dispersive effects in MWCNTs. Intershell tunneling will not be considered in this work.

CNTs have also been considered to constitute nanoantennas. Their performances have been deeply analyzed in [19]-[24]. It has been proven that CNTs offer control of the polarization state, radiation pattern and gain. In the Terahertz frequency range, quantum mechanical conductivity derived in [19] needs to be considered, since it accounts for interband transitions ignored in the semiclassical analysis. In this paper, the analysis will be restricted up to $1 \mathrm{THz}$ so that the semiclassical conductivity can be considered a good approximation of the full quantum conductivity [25].

When the number of SWCNTs in each bundle increases and the length of the interconnect exceeds some tens of microns, the standard techniques based on the MTL theory suffer from model complexity ad accuracy limitations. To mitigate the problem of the complexity of the overall MTL model related to the increasing number of nanotubes, approximate formulations have been proposed based on the equivalent single conductor (ESC) distributed circuit in the frequency-domain [16], [26][28]. Unfortunately, the approximations used to speed-up the simulation time leads to loss of accuracy especially in the case 
of long interconnects for frequencies exceeding some tens of $\mathrm{GHz}$ [28]. Also, the intrinsic frequency-domain formulation does not allow a seamless integration of the interconnect model with non-linear drivers and receivers, which is fundamental for signal integrity and crosstalk analysis. Recently, a novel technique for SWCNTs has been proposed in [29], which allows overcoming all the limits exhibited by the other techniques. It is able to construct accurate and efficient models of large SWCNTs interconnects, while system properties such as stability and passivity are guaranteed for a suitable timedomain analysis (e.g. in time-domain circuit simulator such as SPICE-like solvers [30]) needed for signal integrity and crosstalk analysis. This method can be extended to MWCNTs and MWCNTs bundles, considering that the only difference relies on the computation of the per-unit-length (p.u.l.) parameters [16], [31].

During the design of nanointerconnects it is very important to predict the response of the circuit under study as a function of design parameters, such as fluctuations in the critical dimensions of the layout. A typical design process includes design space exploration, optimization, variability analysis, and therefore it requires repeated simulations for different design parameter values (see Fig. 1).

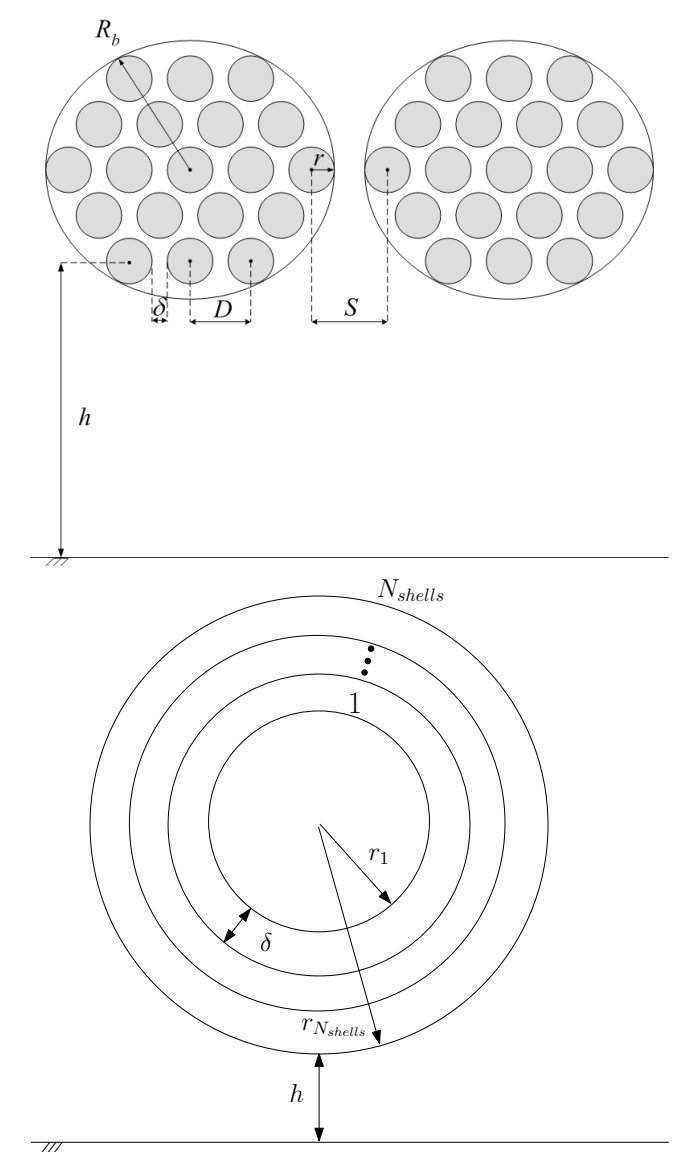

Fig. 1. Geometric configuration of two SWCNT bundles and an MWCNT above a ground plane.

Using MTL-based solvers to perform these design activities is not an efficient choice, since each MTL-based simulation may be expensive for large SWCNTs and MWCNTs. A recent method [31] proposes to model the relation between the time delay at $50 \%$ of MWCNT-based interconnects and some design parameters using a polynomial model that interpolates the results of numerical simulations performed applying the ESC model for an MWCNT. This polynomial model is then used in an optimization process to identify the minimum number of shells of an MWCNT required to guarantee a time delay at $50 \%$ lower than that one associated with a conventional copper-based nanointerconnect.

Parametric macromodels can be used to accurately and efficiently model the effects of design parameters variations on the system behavior, avoiding the brute-force use of MTLbased solvers. These models are able to describe the complex behavior of SWCNTs and MWCNTs at the input/output ports (e.g. scattering, admittance and impedance input-output representations) as a function of frequency (time) and additional design parameters. Therefore, parametric macromodels are more general than the approach in [31] and can be used for several design activities (e.g. design space exploration, optimization, variability analysis). The accuracy, compactness and preservation of system properties (e.g. stability and passivity) are fundamental and required features of parametric macromodels. Over the years, some parametric macromodeling techniques able to guarantee stability and passivity over the design space of interest have been proposed [32]-[37].

The parametric macromodeling method proposed in [37] generates accurate parametric macromodels whose size is not affected by the number of design parameters in addition to frequency. It leads to efficient and compact models. Stability and passivity are preserved over the design space of interest. This technique is based on an innovative interpolation approach based on positive interpolation operators, frequency scaling coefficients and linear least-square problems.

This paper proposes a parametric macromodeling method for the efficient design of CNT-based interconnects (SWCNTs and MWCNTs). First, a set of frequency-domain data samples of a compact admittance representation are computed using the method [29] over a set of design parameter values. Then, a parametric macromodel is built using these initial data by means of the algorithm described [37] to accurately and efficient describe the effect of design parameters on the behavior of nanointerconnects.

The paper is organized as follows: Section II recalls the modeling method [29] to get a compact admittance representation of SWCNTs and MWCNTs systems. Section III proposes a parametric macromodeling method for SWCNTs and MWCNTs structures. Pertinent numerical results in frequency- and time-domain validate the proposed methodology in Section IV. The conclusions are drawn in Section V.

\section{Modeling of SWCNTS AND MWCNTS}

This section briefly describes the method presented in [29] and extends it for MWCNTs. A more detailed description can be found in [29] and it is not repeated here. Consider a nanointerconnect constituted by $N_{b}$ bundles, where each bundle is constituted by $N_{c}^{b}$ SWCNTs, so that the global number of nanotubes is $N_{c}=N_{b} N_{c}^{b}$. Once the impedance 
matrix $Z$ is obtained through the exact MTL theory [38] or modal decompositon [39] starting from p.u.l. parameters, the additional resistance $R_{t}$ of a metallic SWCNT can be incorporated [29]:

$$
\boldsymbol{Z}_{t}(s)=\boldsymbol{Z}(s)+\left[\operatorname{diag}\left(R_{t}\right)\right]_{2 N_{c} \times 2 N_{c}}
$$

The global impedance matrix $\boldsymbol{Z}_{t}(s)$ can be converted into the admittance form $\boldsymbol{Y}_{t}(s)$ in the frequency domain as:

$$
\boldsymbol{Y}_{t}\left(j \omega_{l}\right)=\boldsymbol{Z}_{t}^{-1}\left(j \omega_{l}\right), \quad \omega_{l}=2 \pi f_{l}, l=1, \cdots, N_{f}
$$

where $N_{f}$ denotes the number of frequency samples. Two conditions can be enforced based on the observation that, within each bundle, the nanotubes are connected in parallel:

1) the port voltage is common to all nanotubes within each bundle

$$
\begin{aligned}
& \boldsymbol{V}_{0}\left(j \omega_{l}\right)=\boldsymbol{V}_{0, i}\left(j \omega_{l}\right) \quad i=1, \cdots, N_{c}^{b} \\
& \boldsymbol{V}_{\ell}\left(j \omega_{l}\right)=\boldsymbol{V}_{\ell, i}\left(j \omega_{l}\right) \quad i=1, \cdots, N_{c}^{b}
\end{aligned}
$$

2) the global current at the input and output port of each bundle is the sum of the currents through each nanotubes within each bundle

$$
\begin{aligned}
& \boldsymbol{I}_{0}\left(j \omega_{l}\right)=\sum_{i=1}^{N_{c}^{b}} \boldsymbol{I}_{0, i}\left(j \omega_{l}\right) \quad i=1, \cdots, N_{c}^{b} \\
& \boldsymbol{I}_{\ell}\left(j \omega_{l}\right)=\sum_{i=1}^{N_{c}^{b}} \boldsymbol{I}_{\ell, i}\left(j \omega_{l}\right) \quad i=1, \cdots, N_{c}^{b}
\end{aligned}
$$

for each frequency sample $\omega_{l}$. Hence, the $N_{b}$ bundles can be represented as a $2 N_{b}$ port system whose admittance matrix representation, for each frequency sample, reads:

$$
\left[\begin{array}{l}
\boldsymbol{I}_{0}\left(j \omega_{l}\right) \\
\boldsymbol{I}_{\ell}\left(j \omega_{l}\right)
\end{array}\right]=\left[\begin{array}{ll}
\widetilde{\boldsymbol{Y}}_{t, 11}\left(j \omega_{l}\right) & \widetilde{\boldsymbol{Y}}_{t, 12}\left(j \omega_{l}\right) \\
\widetilde{\boldsymbol{Y}}_{t, 21}\left(j \omega_{l}\right) & \widetilde{\boldsymbol{Y}}_{t, 22}\left(j \omega_{l}\right)
\end{array}\right]\left[\begin{array}{l}
\boldsymbol{V}_{0}\left(j \omega_{l}\right) \\
\boldsymbol{V}_{\ell}\left(j \omega_{l}\right)
\end{array}\right],
$$

where each sub-block $\tilde{\boldsymbol{Y}}_{t, m n}\left(j \omega_{l}\right), m, n=1,2$ is obtained summing the entries of a order- $N_{c}^{b}$ sub-block of the matrix $\boldsymbol{Y}_{t}\left(j \omega_{l}\right)(2)$.

A time-domain macromodel can easily be obtained by applying the Vector Fitting (VF) technique [40] to the admittance matrix (5):

$$
\tilde{\boldsymbol{Y}}_{t}(s)=\mathbf{C}_{0}+\sum_{n=1}^{N} \frac{\mathbf{C}_{n}}{s-p_{n}} .
$$

It is to be noted that the fitting process is extremely fast since it is applied to a reduced number of ports. The stability of a rational macromodel obtained by means of the VF method is ensured by a simple pole-flipping scheme [40], while its passivity [41] is not guaranteed and must be checked and enforced by means of standard techniques (see e.g. [42], [43]). Stability and passivity are fundamental for carrying out stable time-domain analysis (e.g. in time-domain circuit simulator such as SPICE-like solvers [30]).

The corresponding state-space form for the rational macromodel can be written as:

$$
\begin{aligned}
\dot{\boldsymbol{x}}(t) & =\mathcal{A} \boldsymbol{x}(t)+\mathcal{B} \boldsymbol{v}(t), \\
\boldsymbol{i}(t) & =\mathcal{C} \boldsymbol{x}(t)+\mathcal{D} \boldsymbol{v}(t),
\end{aligned}
$$

using standard realization methods [44], [45], where $\mathcal{A} \in$ $\Re^{p \times p}, \mathcal{B} \in \Re^{p \times P_{s y s}}, \mathcal{C} \in \Re^{P_{s y s} \times p}, \mathcal{D} \in \Re^{P_{s y s} \times P_{s y s}}, p$ is the number of states, $P_{\text {sys }}$ denote the number of ports of the system. In the case under analysis, the input and output vectors correspond to port voltages $\boldsymbol{v}(t)$ and currents $\boldsymbol{i}(t)$, respectively.

Each bundle is assumed to be terminated on current sources and voltage-driven lumped linear and nonlinear elements, which can be described by the following equation:

$$
\boldsymbol{i}(t)=\boldsymbol{i}_{s}(t)-\boldsymbol{G}_{t e r m} \boldsymbol{v}(t)-\boldsymbol{C}_{t e r m} \frac{d \boldsymbol{v}(t)}{d t}-\boldsymbol{f}(\boldsymbol{v}(t)),
$$

where $\boldsymbol{v}(t)$ and $\boldsymbol{i}(t)$ are the port voltages and currents, the matrices $\boldsymbol{G}_{\text {term }}, \boldsymbol{C}_{\text {term }}$ model linear resistive and capacitive terminations, respectively, and $\boldsymbol{f}(\boldsymbol{v}(t))$ describes lumped nonlinear components. The global nonlinear system (7)-(8) can be solved by using standard techniques [46].

This modeling method can be extended to MWCNTs and MWCNTs bundles, considering that the only difference relies on the computation of the p.u.l. parameters [16], [31].

\section{PARAMETRIC MACROMOdeling OF SWCNTS AND MWCNTS}

In Section II, we have discussed how to create a compact admittance matrix (5) for interconnects composed of bundles of SWCNTs and MWCNTs using the approach in [29]. This representation only depends on the Laplace variable $s$. If design parameters $\mathbf{g}=\left(\mathrm{g}^{(m)}\right)_{m=1}^{M}$ (such as layout features (e.g. $S, D, r, R_{b}, h$ in Fig. 1)) must be taken into account in the modeling process, then parametric macromodels can be used to build a multidimensional representation of $\widetilde{\mathbf{Y}}_{t}(s, \mathbf{g})$.

The parametric macromodeling method described in [37] is able to build a parametric macromodel in the form:

$$
\mathbf{R}(s, \mathbf{g})=\mathbf{C}_{0}(\mathbf{g})+\sum_{n=1}^{N(\mathbf{g})} \frac{\mathbf{C}_{n}(\mathbf{g})}{s-p_{n}(\mathbf{g})}
$$

or equivalently

$$
\mathbf{R}(s, \mathbf{g})=\mathcal{C}(\mathbf{g})(s \mathcal{I}-\mathcal{A}(\mathbf{g}))^{-1} \mathcal{B}(\mathbf{g})+\mathcal{D}(\mathbf{g})
$$

starting from a set of multivariate data samples $\left\{\left(s_{l}, \mathbf{g}_{k}\right), \widetilde{\mathbf{Y}}_{t}\left(s_{l}, \mathbf{g}_{k}\right)\right\}, l=1, \ldots, N_{f}, k=1, \ldots, K_{t o t}$ which depend on the complex frequency $s=j \omega$ and $\mathbf{g}$. Two design space data grids are used in the modeling process: an estimation grid and a validation grid. The estimation grid is utilized to build a parametric macromodel. The validation grid is used to validate the modeling capability of the parametric macromodel in a set of points of the design space previously not used for its construction. The design space contains all parameters g. To clarify the use of these two design space grids, we show in Fig. 2 a possible estimation and validation design space grid in the case of two design parameters $\mathbf{g}=\left(\mathrm{g}^{(1)}, \mathrm{g}^{(2)}\right)$. 


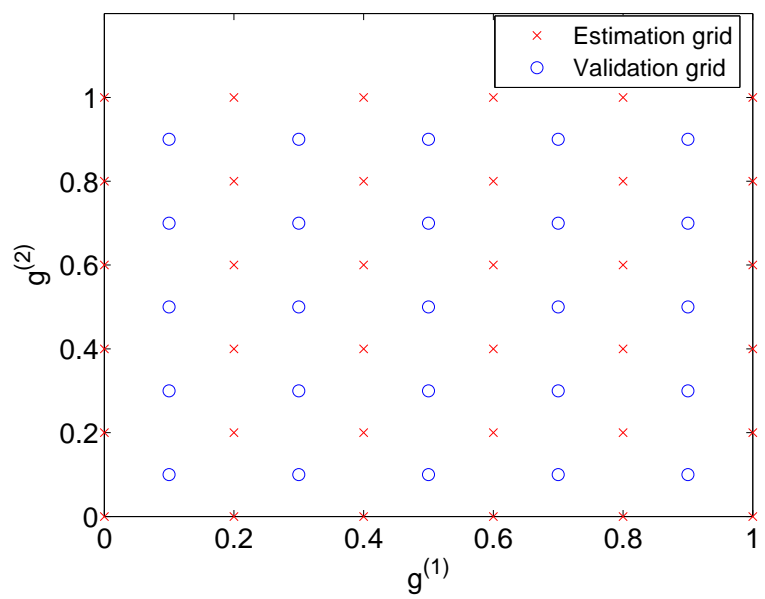

Fig. 2. An example of estimation and validation design space grid.

As a first step in [37], the design space is divided into cells: hyperrectangles (regular grids) [47] or simplices (regular and scattered grids) [48]. We indicate a cell region of the design space as $\Omega_{i}, i=1, \ldots, P$ and the corresponding vertices as $\mathbf{g}_{k}^{\Omega_{i}}, \quad k=1, \ldots, Q$. For each cell a reference macromodel is computed in the form

$$
\mathbf{R}\left(s, \mathbf{g}_{k}^{\Omega_{i}}\right)=\mathbf{C}_{0}\left(\mathbf{g}_{k}^{\Omega_{i}}\right)+\sum_{n=1}^{N\left(\Omega_{i}\right)} \frac{\mathbf{C}_{n}\left(\mathbf{g}_{k}^{\Omega_{i}}\right)}{s \alpha\left(\mathbf{g}_{k}^{\Omega_{i}}\right)-p_{r e f, n}\left(\Omega_{i}\right)}
$$

using the data samples $\widetilde{\mathbf{Y}}_{t}\left(s, \mathbf{g}_{k}^{\Omega_{i}}\right)$, the VF method, optimization steps, linear least-squares solutions and passivity enforcement techniques [37]. Each design space cell is described by its reference poles $p_{\text {ref, } n}\left(\Omega_{i}\right)$, vertex frequency scaling coefficients, residues and direct terms $\alpha\left(\mathbf{g}_{k}^{\Omega_{i}}\right), \mathbf{C}_{n}\left(\mathbf{g}_{k}^{\Omega_{i}}\right), \mathbf{C}_{0}\left(\mathbf{g}_{k}^{\Omega_{i}}\right)$, $k=1, \ldots, Q$. The reference poles are computed at one cell vertex (reference point) for which $\alpha=1$.

The next step is building a parametric macromodel $\mathbf{R}(s, \mathbf{g})$ using suitable interpolation schemes that are applied to vertex frequency scaling coefficients, residues and direct terms for each design space cell. If the response of the system under modeling needs to be computed at a specific point $\widehat{\mathbf{g}} \widehat{ }$ of a design space cell $\widehat{\Omega}$, the frequency scaling coefficients $\alpha\left(\mathbf{g}_{k}^{\widehat{\Omega}}\right)$, residues $\mathbf{C}_{n}\left(\mathbf{g}_{k}^{\widehat{\Omega}}\right)$, direct terms $\mathbf{C}_{0}\left(\mathbf{g}_{k}^{\widehat{\Omega}}\right)$ are interpolated in $\widehat{\mathbf{g}} \widehat{\Omega}$ and a rational model

$$
\mathbf{R}\left(s, \widehat{\mathbf{g}}^{\widehat{\Omega}}\right)=\mathbf{C}_{0}\left(\widehat{\mathbf{g}}^{\widehat{\Omega}}\right)+\sum_{n=1}^{N(\widehat{\Omega})} \frac{\mathbf{C}_{n}\left(\widehat{\mathbf{g}}^{\widehat{\Omega}}\right)}{s \alpha\left(\widehat{\mathbf{g}}^{\widehat{\Omega}}\right)-\mathbf{p}_{r e f, n}(\widehat{\Omega})}
$$

is obtained [37].

It is important to highlights that the method described in [37] is able to generate parametric macromodels whose size is not increasing with the number of design parameters, therefore the model compactness for high-dimensional design spaces is preserved. Stability and passivity are preserved over the entire design space of interest, which allows performing stable time-domain simulations for different values of the design parameters.

Frequency-domain data samples of the compact admittance matrix (5) corresponding to estimation and validation design space points are the estimation and validation data used to build and validate a parametric macromodel. To generate those data samples over the estimation and validation design space points, an initial computational effort is needed to compute the impedance matrix $Z$ through the exact MTL theory [38] or modal decompositon [39] and then to apply the method [29]. Once the parametric macromodel is created and validated, it becomes an accurate and efficient surrogate of the original system and can be used for each related design space exploration, optimization and variability analysis in the design space defined during the construction of the parametric macromodel. Multiple uses of the parametric macromodel in design activities makes the corresponding computational effort for the model generation negligible.

For a parametric macromodel (9)-(10), the corresponding parametric state-space equivalent form can be written as:

$$
\begin{aligned}
\dot{\boldsymbol{x}}(t, \mathbf{g}) & =\mathcal{A}(\mathbf{g}) \boldsymbol{x}(t, \mathbf{g})+\mathcal{B}(\mathbf{g}) \boldsymbol{v}(t, \mathbf{g}), \\
\boldsymbol{i}(t, \mathbf{g}) & =\mathcal{C}(\mathbf{g}) \boldsymbol{x}(t, \mathbf{g})+\mathcal{D}(\mathbf{g}) \boldsymbol{v}(t, \mathbf{g}),
\end{aligned}
$$

with termination equations:

$\boldsymbol{i}(t, \mathbf{g})=\boldsymbol{i}_{s}(t)-\mathbf{g}_{t e r m} \boldsymbol{v}(t, \mathbf{g})-\boldsymbol{C}_{t e r m} \frac{d \boldsymbol{v}(t, \mathbf{g})}{d t}-\boldsymbol{f}(\boldsymbol{v}(t, \mathbf{g}))$.

\section{NUMERICAL RESULTS}

In what follows, two pertinent examples have been considered with frequency- and time-domain numerical results parameterized as a function of suitable design parameters. The SWCNTs are assumed to be all conductive within each bundle and semiconducting tubes are neglected [16].

The results of the proposed parametric macromodel approach in time-domain are compared with those obtained by applying the inverse fast Fourier transform (IFFT) to the frequency-domain spectra of port currents and voltages computed by the rigorous transmission line theory and a proper combination of $\boldsymbol{Y}_{t}(s)$ and terminations. The time-domain integration of the parametric macromodels is carried out by means of the Backward Euler algorithm [49].

The worst case of the error function:

$$
\begin{aligned}
& \operatorname{Err}(\mathbf{R}(s, \mathbf{g}), \mathbf{H}(s, \mathbf{g}))= \\
& =\max _{i, j} \sqrt{\frac{1}{N_{f}}} \sqrt{\sum_{l=1}^{N_{f}} \frac{\left|R_{(i, j)}\left(s_{l}, \mathbf{g}\right)-H_{(i, j)}\left(s_{l}, \mathbf{g}\right)\right|^{2}}{\left|\max _{l}\left(H_{(i, j)}\left(s_{l}, \mathbf{g}\right)\right)\right|^{2}}} \\
& i=1, \ldots, P_{\text {sys }}, j=1, \ldots, P_{\text {sys }}
\end{aligned}
$$

over the validation grid is chosen to assess the accuracy and quality of parametric macromodels:

$$
\mathbf{g}_{\max }=\underset{\mathbf{g} \in \text { validation grid }}{\operatorname{argmax}} \operatorname{Err}(\mathbf{R}(s, \mathbf{g}), \mathbf{H}(s, \mathbf{g}))
$$




$$
\operatorname{Err}_{\max }=\operatorname{Err}\left(\mathbf{g}_{\max }\right)
$$

$\widetilde{\boldsymbol{Y}}_{t}$ here $\mathbf{H}(s, \mathbf{g})$ is $\widetilde{\boldsymbol{Y}}_{t}(s, \mathbf{g})$. In the numerical results, we denote $\tilde{\boldsymbol{Y}}_{t}$ as $\boldsymbol{Y}$ for ease of notation.

All numerical experiments were performed using Matlab R2009A [50] and on Windows platform equipped with Intel Core2 Extreme CPU Q9300 $2.53 \mathrm{GHz}$ and 8 GB RAM.

\section{A. 3D model of an MWCNT}

In this first example, an MWCNT of 4 shells is considered. The radius of the $\mathrm{n}$-th shell is given by $r_{n}=r+(n-1) \delta$ with $\delta=0.34 \mathrm{~nm}$. Three design parameters are considered in addition to frequency, namely the height above the reference plane $h$, the radius of the first shell $r$ and the length $L$ of the interconnect. Table I shows their corresponding ranges.

TABLE I

DESIGN PARAMETERS.

\begin{tabular}{|c|c|c|}
\hline Parameter & Min & Max \\
\hline Frequency (freq) & $1 \mathrm{kHz}$ & $1 \mathrm{THz}$ \\
\hline Hei & $35 \mathrm{~nm}$ & $65 \mathrm{~nm}$ \\
\hline $\operatorname{Rad}$ & $5 \mathrm{~nm}$ & $20 \mathrm{~nm}$ \\
\hline Length (L) & $0.5 \mu \mathrm{m}$ & $1.5 \mu \mathrm{m}$ \\
\hline
\end{tabular}

The technique [29] has been used to evaluate the admittance parameters $\mathbf{Y}(s, h, r, L)$ for 201 logarithmically spaced frequency samples over an estimation grid of $4 \times 4 \times 4(h, r, L)$ samples and a validation grid of $3 \times 3 \times 3(h, r, L)$ samples. The method [37] is used to build a 4-D model using a multilinear interpolation scheme in the parameterization steps. The error defined in (17) is equal to 0.0056 .

Figs. 3-4 show the magnitude of the parametric macromodel of $\mathbf{Y}_{11}(s, h, r, L)$ for $h=50 \mathrm{~nm}, r=12.5 \mathrm{~nm}$ and of $\mathbf{Y}_{12}(s, h, r, L)$ for $h=50 \mathrm{~nm}, L=1 \mu \mathrm{m}$. Fig. 5 compares $\mathbf{Y}_{11}(s, h, r, L)$ and its model for the validation values $h=50$ $\mathrm{nm}, r=12.5 \mathrm{~nm}, L=\{0.67,1.33\} \mathrm{nm}$. These results show that the parametric macromodel is able to accurately describe the system behavior as a function of frequency and multiple design parameters. The CPU time needed to compute one frequency sample using the parametric macromodel is equal to $97 \mu$ s, which confirms the high model efficiency for design activities that require multiple simulations.

\section{B. 2D model of four bundles of SWCNTs}

In this second example, four parallel bundles of SWCNTs, each of 52 nanotubes, are considered. The inter-tube distance is $\delta=0.34 \mathrm{~nm}$, the lattice constant is $D=1.14 \mathrm{~nm}$ and the spacing between the bundles is $S=25 \mathrm{~nm}$. The length of the bundles is $L=30 \mu \mathrm{m}$. We consider two design parameters in addition to frequency, namely the height above the reference plane $(h)$ and the radius of each nanotube $(r)$. Their corresponding ranges are shown in Table II.

The admittance parameters $\mathbf{Y}(s, h, r)$ have been computed by means of the method [29] for 201 logarithmically spaced frequency sample over an estimation grid of $5 \times 5(h, r)$

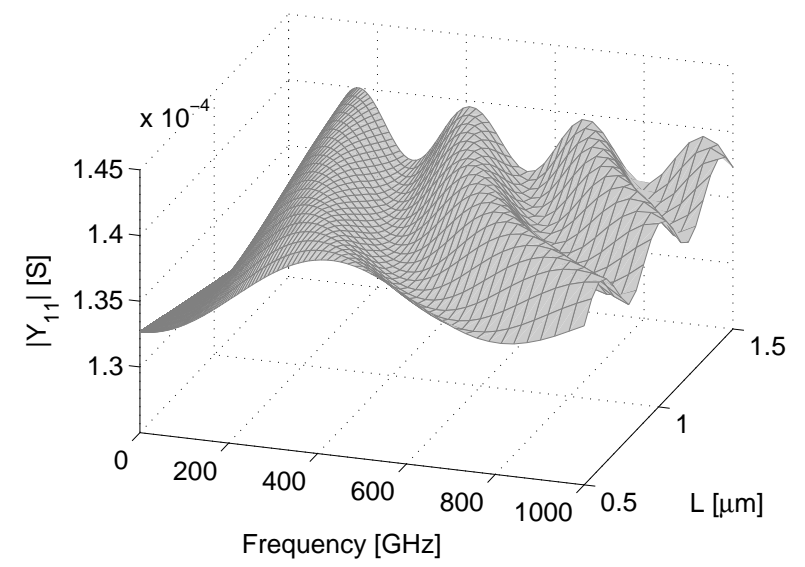

Fig. 3. Magnitude of the 4-D model of $\mathbf{Y}_{11}(s, h, r, L)(h=50 \mathrm{~nm}$, $r=12.5 \mathrm{~nm})$.

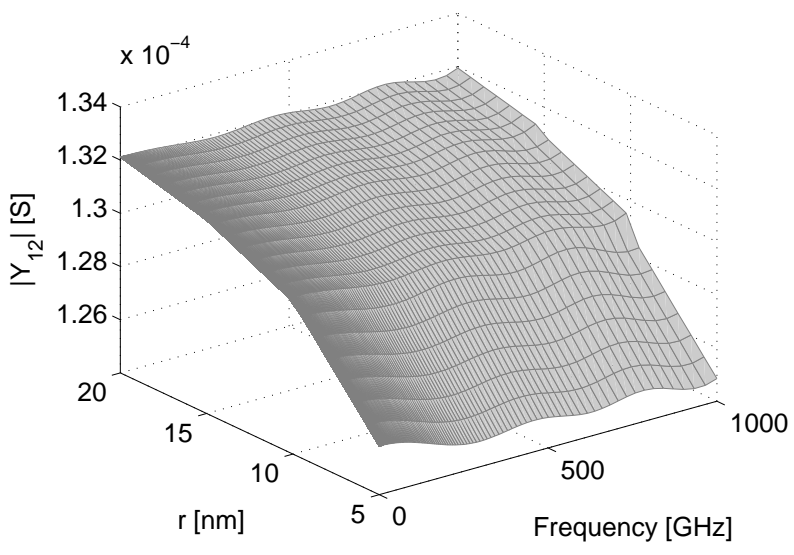

Fig. 4. Magnitude of the 4-D model of $\mathbf{Y}_{12}(s, h, r, L)(h=50 \mathrm{~nm}, L=1$ $\mu \mathrm{m})$.

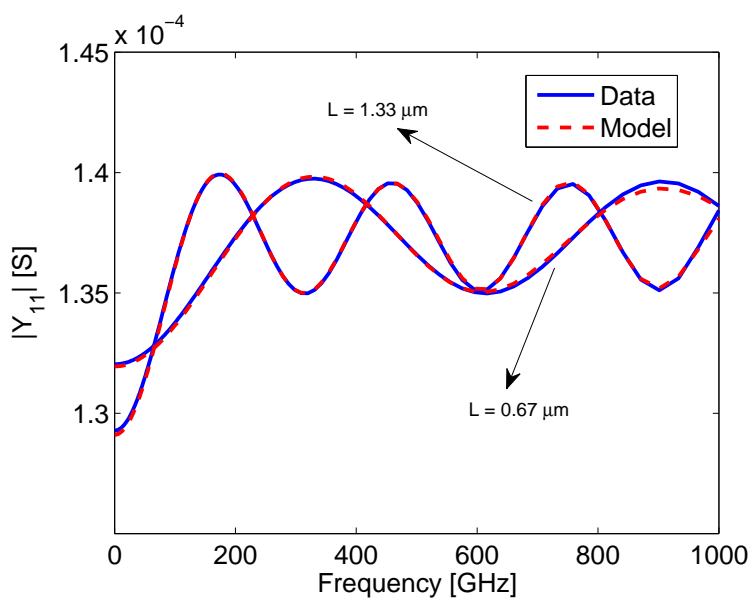

Fig. 5. Magnitude of $\mathbf{Y}_{11}(s, h, r, L)(h=50 \mathrm{~nm}, r=12.5 \mathrm{~nm}, L=$ $\{0.67,1.33\} \mu \mathrm{m})$.

samples and a validation grid of $4 \times 4(h, r)$ samples. The method [37] is used to build a 3-D model. The multilinear 
TABLE II

DESIGN PARAMETERS.

\begin{tabular}{|l|c|c|}
\hline Parameter & Min & Max \\
\hline Frequency (freq) & $1 \mathrm{kHz}$ & $1 \mathrm{THz}$ \\
Height (h) & $70 \mathrm{~nm}$ & $140 \mathrm{~nm}$ \\
Radius (r) & $0.4 \mathrm{~nm}$ & $0.9 \mathrm{~nm}$ \\
\hline
\end{tabular}

interpolation scheme is used in the parameterization steps. The error defined in (17) is equal to 0.0038 .

Figs. 6-7 show the magnitude of the parametric macromodel of $\mathbf{Y}_{11}(s, h, r)$ for $h=96.25 \mathrm{~nm}$ and of $\mathbf{Y}_{18}(s, h, r)$ for $r=0.5875 \mathrm{~nm}$. Fig. 8 compares $\mathbf{Y}_{11}(s, h, r)$ and its model for the validation values $r=\{0.4625,0.5875,0.7125,0.8375\}$ $\mathrm{nm}, h=96.25 \mathrm{~nm}$. A good agreement between the model and data can be observed over the design space of interest. As in the previous example, the model is very efficient to be evaluated and the CPU time needed to compute one frequency sample is equal to $148 \mu \mathrm{s}$.

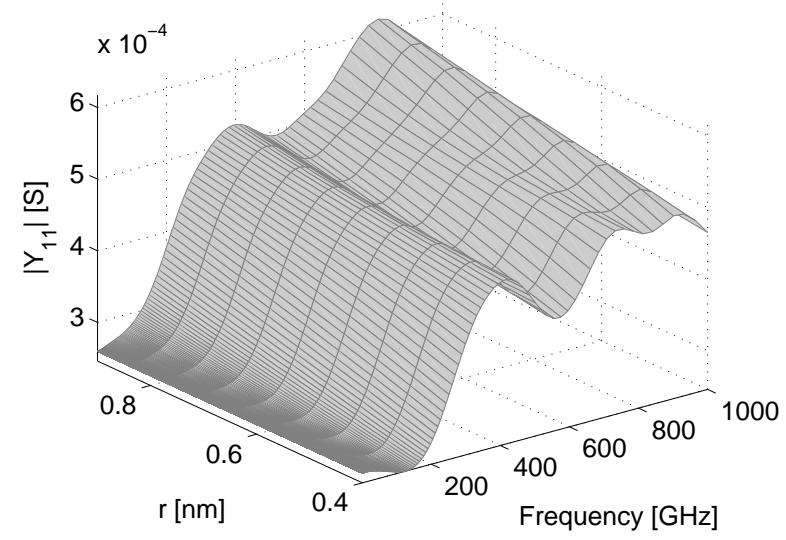

Fig. 6. Magnitude of the 3-D model of $\mathbf{Y}_{11}(s, h, r)(h=96.25 \mathrm{~nm})$.

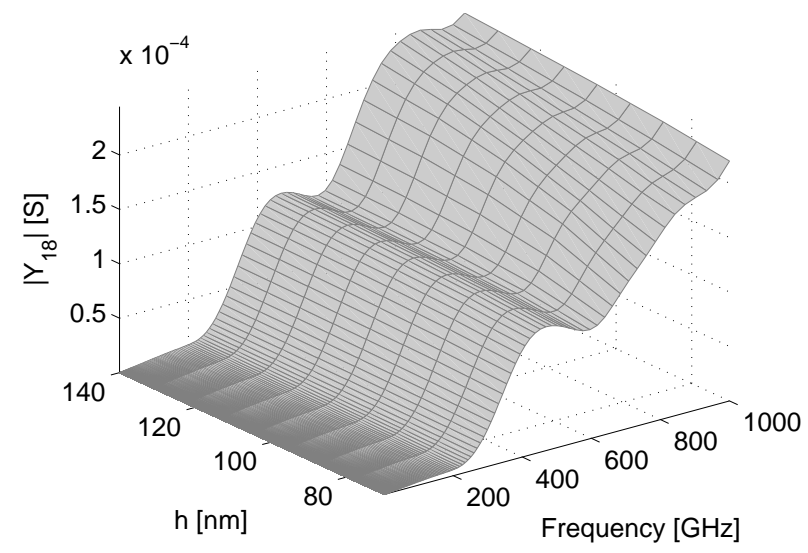

Fig. 7. Magnitude of the 3-D model of $\mathbf{Y}_{18}(s, h, r)(r=0.5875 \mathrm{~nm})$.

Since the parametric macromodel is stable and passive over the design space, some time-domain simulations have been

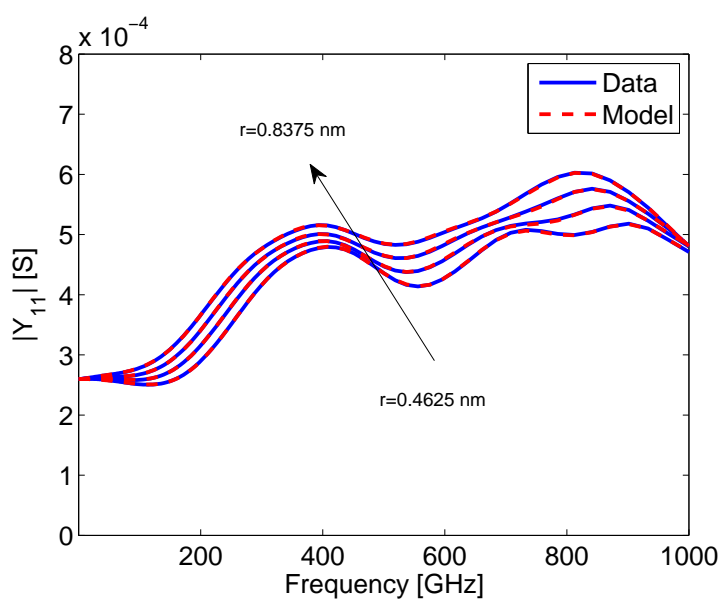

Fig. 8. Magnitude of $\mathbf{Y}_{11}(s, h, r)(r=\{0.4625,0.5875,0.7125,0.8375\}$ $\mathrm{nm}, h=96.25 \mathrm{~nm})$.

performed. The first bundle is excited at the input port by two voltage pulses with amplitude $100 \mathrm{mV}$, rise time $\tau_{r}=1 \mathrm{ps}$ and pulse width equal to $20 \mathrm{ps}$ (see Fig. 9). All input ports are terminated by a resistance equal to $15 \mathrm{k} \Omega$, while the output ports are terminated by a capacitance equal to $0.1 \mathrm{fF}$.

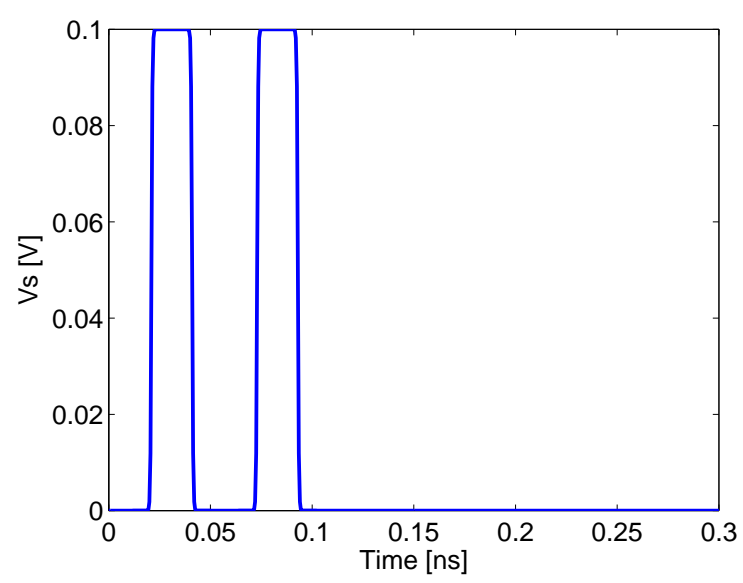

Fig. 9. Voltage source.

Fig. 10 shows the transient voltage at the input port closest to the voltage source (near-end crosstalk). The model results are in a good agreement with those of the IFFT.

These numerical results show the accuracy of the parametric macromodel in both frequency- and time-domain. The capability of the model to guarantee overall stability and passivity results in efficient and stable parameterized timedomain simulations. 

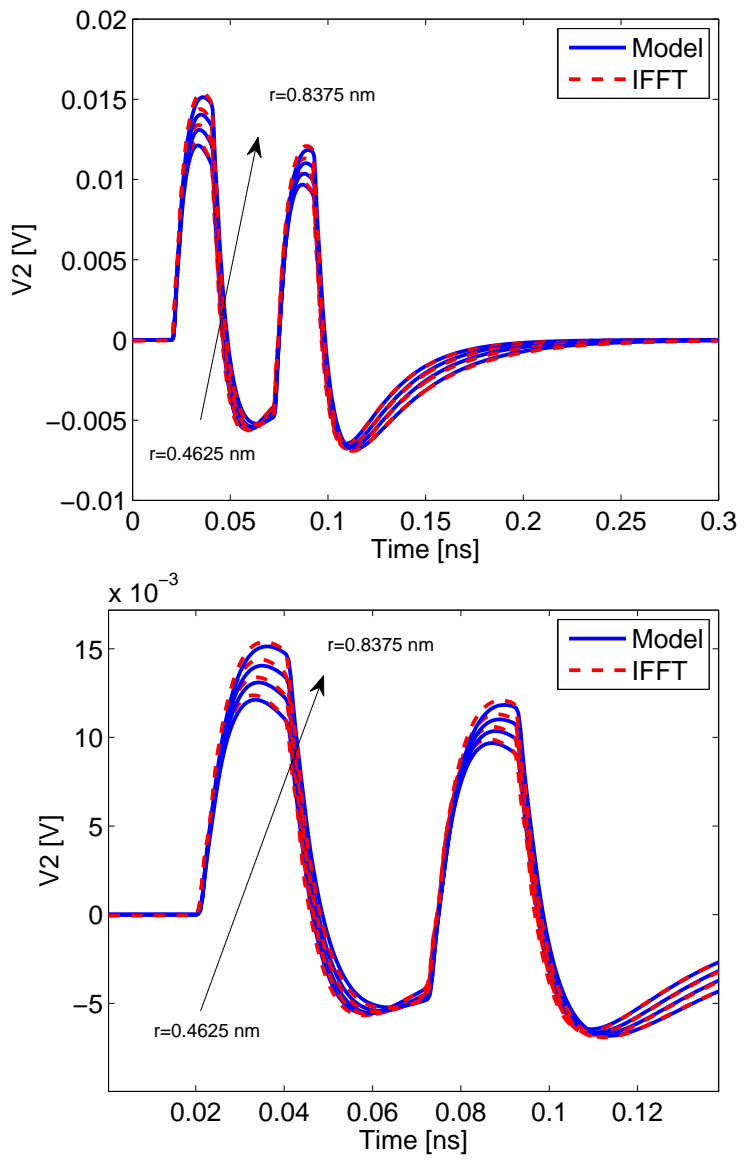

Fig. 10. Near-end crosstalk voltage $(r=\{0.4625,0.5875,0.7125,0.8375\}$ $\mathrm{nm}, h=96.25 \mathrm{~nm})$.

\section{CONCLUSIONS}

We have proposed an efficient and robust methodology to study the effect of design parameters variations on the performances of CNT-based interconnects by means of parametric macromodels. This work presents novel design tools that can provide significant speed-ups avoiding the brute-force use of MTL-based solvers, while maintaining a high accuracy. Pertinent examples have been considered with parameterized frequency- and time-domain numerical results to validate the proposed approach.

\section{REFERENCES}

[1] P. L. McEuen, M. S. Fuhrer and H. Park, "Single-walled carbon nanotube electronics," IEEE Transactions on Nanotechnology, vol. 1, no. 1, pp. 78-85, Mar. 2002.

[2] A. Naeemi, R. Sarvari and J. D. Meindl, "Performance comparison between carbon nanotube and copper interconnects for gigascale integration GSI," IEEE Electron Device Letters, vol. 26, no. 2, pp. 84-86, Feb 2005.

[3] A. Naeemi and J. D. Meindl, "Impact of electron-phonon scattering on the performance of carbon nanotube interconnects for gigascale integration (GSI)," IEEE Transactions on Electron Devices, vol. 27, no. 5, pp. 338-440, May 2006.

[4] A. Raychowdhury and K. Roy, "Modelling of metallic carbon-nanotube interconnects for circuit simulations and a comparison with cu interconnects for scaled technologies," IEEE Transactions on Computer-Aided Design of Integrated Circuits and Systems, vol. 25, no. 1, pp. 58-65, Jan 2006.
[5] A. Naeemi and J. D. Meindl, "Design and performance modelling for single-walled carbon nanotubes as local, semiglobal and global interconnects in gigascale integrated systems," IEEE Transactions on Electron Devices, vol. 54, no. 1, pp. 26-37, Jan. 2007.

[6] J. Li et al., "Bottom-up approach for carbon nanotubes interconnects," Appl. Phys. Lett., vol. 82, pp. 2491-2493, Apr. 2003.

[7] N. Srivastava and K. Banerjee, "Performance analysis of carbon nanotube interconnects for VLSI applications," in Proc. IEEE/ACM ICCAD, 2005, pp. 383-390.

[8] H. Li, W. Y. Yin, K. Banerjee, and J. F. Mao, "Circuit modeling and performance analysis of multi-walled carbon nanotube interconnects," IEEE Transactions on Electron Devices, vol. 55, no. 6, pp. 1328-1337, Jun. 2008.

[9] K. N. Srivastava, H. Li, F. Kreupl, and K. Banerjee, "On the applicability of single-walled carbon nanotubes as VLSI interconnects," IEEE Transactions on Nanotechnology, vol. 8, no. 4, pp. 542-559, Jul. 2009.

[10] W.-S. Zhao, W.-Y. Yin, and Y.-X. Guo, "Electromagnetic compatibilityoriented study on through silicon single-walled carbon nanotube bundle via (TS-SWCNTBV) arrays," IEEE Transactions on Electromagnetic Compatibility, vol. 54, no. 1, pp. 149-157, Feb. 2012.

[11] A. Chiariello, A. Maffucci, and G. Miano, "Electrical modeling of carbon nanotube vias," IEEE Transactions on Electromagnetic Compatibility, vol. 54, no. 1, pp. 158-166, Feb. 2012.

[12] M. S. Sarto and A. Tamburrano, "Multiconductor transmission line modeling of SWCNT bundles in common-mode excitation," in IEEE International Symposium on Electromagnetic Compatibility, Portland, OR, Aug. 2006, pp. 466-471.

[13] M. D'Amore, M. S. Sarto and A. Tamburrano, "Signal integrity of carbon nanotube bundles," in IEEE International Symposium on Elec tromagnetic Compatibility, Honolulu, Hawaii, Jul. 2007.

[14] M. S. Sarto, A. Tamburrano and M. D'Amore, "New electronwaveguide-based modeling for carbon nanotube interconnects," IEEE Transactions on Nanotechnology, vol. 8, no. 2, pp. 214 - 225, Mar. 2009.

[15] S. N. Pu, W. Y. Yin, J. F. Mao and Q. H. Liu, "Crosstalk prediction of single- and double-walled carbon-nanotube (SWCNT/DWCNT) bundle interconnects," IEEE Transactions on Electron Devices, vol. 56, no. 4, pp. 560-568, Apr. 2009.

[16] M. D'Amore, A. Tamburrano and M. S. Sarto, "Fast transient analysis of next-generation interconnects based on carbon nanotubes," IEEE Transactions on Electromagnetic Compatibility, vol. 52, no. 2, pp. 496 - 503, May 2010.

[17] C. Forestiere, A. Maffucci, S. Maksimenko, G. Miano, and G. Y. Slepyan, "Transmission-line model for multiwall carbon nanotubes with intershell tunneling," IEEE Transactions on Nanotechnology, vol. 11, no. 3, pp. 554-564, May 2012.

[18] A. Maffucci and G. Miano, "A general transmission line model for conventional metallic nanowires and innovative carbon nano-interconnects," in 17th IEEE Workshop on Signal and Power Integrity (SPI), Paris, France, May 2013, pp. 1-4.

[19] G. Y. Slepyan, S. A. Maksimenko, A. Lakhtakia, O. Yevtushenko, and A. V. Gusakov, "Electrodynamics of carbon nanotubes: Dynamic conductivity, impedance boundary conditions, and surface wave propagation," Phys. Rev. B, vol. 60, pp. 17 136-17 149, Dec 1999.

[20] G. Hanson, "Fundamental transmitting properties of carbon nanotube antennas," IEEE Transactions on Antennas and Propagation, vol. 53, no. 11, pp. 3426-3435, Nov. 2005.

[21] G. Y. Slepyan, M. V. Shuba, S. A. Maksimenko, and A. Lakhtakia, "Theory of optical scattering by achiral carbon nanotubes and their potential as optical nanoantennas," Phys. Rev. B, vol. 73, no. 19, p. 195416, May 2006.

[22] M. V. Shuba, S. A. Maksimenko, and A. Lakhtakia, "Electromagnetic wave propagation in an almost circular bundle of closely packed metallic carbon nanotubes," Phys. Rev. B, vol. 76, no. 15, p. 155407, Oct. 2007.

[23] P. J. Burke, S. Li, and Z. Yu, "Quantitative theory of nanowire and nanotube antenna performance," IEEE Transactions on Nanotechnology, vol. 5, no. 4, pp. 314-334, July 2006.

[24] M. V. Shuba, G. Y. Slepyan, S. A. Maksimenko, C. Thomsen, and A. Lakhtakia, "Theory of multiwall carbon nanotubes as waveguides and antennas in the infrared and the visible regimes," Phys. Rev. B, vol. 79 , no. 15 , p. 155403 , Apr. 2009.

[25] A. Chiariello, C. Forestiere, G. Miano, and A. Maffucci, "Scattering properties of carbon nanotubes," COMPEL: The International Journal for Computation and Mathematics in Electrical and Electronic Engineering, vol. 32, no. 6, pp. 1793 - 1808, 2013.

[26] M. D'Amore, M. S. Sarto and A. Tamburrano, "Transient analysis of crosstalk coupling between high-speed carbon nanotube interconnects," 
in IEEE International Symposium on Electromagnetic Compatibility, Detroit, MI, Aug. 2008, pp. 1-6.

[27] M. D'Amore, M. Ricci and A. Tamburrano, 'Equivalent singleconductor modeling of carbon nanotube bundles for transient analysis of high-speed interconnects," in Proc. of Int. Symp. IEEE-NANO, Arlington, VA, Aug. 2008.

[28] M. S. Sarto, A. Tamburrano and A. D'Aloia, "Equivalent effective p.u.1. parameters for reduced circuit of SWCNT interconnects," in Proc. of Int. Symp. IEEE-NANO, Genoa, Jul. 2009.

[29] F. Ferranti, G. Antonini, T. Dhaene, L. Knockaert, and A. Orlandi, "Compact and accurate models of large single-wall carbon-nanotube interconnects," IEEE Transactions on Electromagnetic Compatibility, vol. 53, no. 4, pp. $1025-1033$, Nov. 2011.

[30] L. W. Nagel, "SPICE: A computer program to simulate semiconductor circuits," University of California, Berkeley, Electr. Res. Lab. Report ERL M520, May 1975.

[31] P. Lamberti, M. Sarto, V. Tucci, and A. Tamburrano, "Robust design of high-speed interconnects based on an MWCNT," IEEE Transactions on Nanotechnology, vol. 11, no. 4, pp. 799 -807, July 2012.

[32] F. Ferranti, L. Knockaert, and T. Dhaene, "Guaranteed passive parameterized admittance-based macromodeling," IEEE Trans. Adv. Packag., vol. 33, no. 3, pp. $623-629$, Aug. 2010

[33] _ - "Parameterized S-parameter based macromodeling with guaranteed passivity," IEEE Microw. Wireless Compon. Lett., vol. 19, no. 10, pp. 608-610, Oct. 2009.

[34] P. Triverio, M. Nakhla, and S. Grivet-Talocia, "Passive parametric macromodeling from sampled frequency data," in IEEE 14th Workshop on Signal Propagation on Interconnects (SPI), 2010, May 2010, pp. 117 -120 .

[35] — , "Passive parametric modeling of interconnects and packaging components from sampled impedance, admittance or scattering data," in Electronic System-Integration Technology Conference (ESTC), 2010, Sept. 2010, pp. 1-6.

[36] F. Ferranti, L. Knockaert, and T. Dhaene, "Passivity-preserving parametric macromodeling by means of scaled and shifted state-space systems," IEEE Trans. Microw. Theory Tech., vol. 59, no. 10, pp. 2394-2403, Oct. 2011.

[37] F. Ferranti, T. Dhaene, and L. Knockaert, "Compact and passive parametric macromodeling using reference macromodels and positive interpolation operators," IEEE Trans. Components, Packaging and Manufacturing Technology, vol. 2, no. 12, pp. 2080 -2088, Dec. 2012.

[38] C. R. Paul, Analysis of Multiconductor Transmission Lines, 2nd ed. New York, NY: John Wiley \& Sons, 2008.

[39] G. Antonini, "A dyadic Green's function based method for the transient analysis of lossy and dispersive multiconductor transmission lines," IEEE Transactions on Microwave Theory and Techniques, vol. 56, no. 4, pp. 880-895, Apr. 2008.

[40] B. Gustavsen and A. Semlyen, "Rational approximation of frequency domain responses by vector fitting," IEEE Transactions on Power Apparatus and Systems, vol. 14, no. 3, pp. 1052-1061, Jul. 1999.

[41] B. D. Anderson, S. Vongpanitlerd, Network Analysis and Synthesis. Englewood Cliffs, NJ, 1973.

[42] D. Saraswat, R. Achar and M. S. Nakhla, "Global passivity enforcement algorithm for macromodels of interconnect subnetworks characterized by tabultaed data," IEEE Transactions on Very Large Scale Integration (VLSI) Systems, vol. 13, no. 7, pp. 819 - 832, Jul. 2005.

[43] B. Gustavsen, "Fast passivity enforcement for pole-residue models by perturbation of residue matrix eigenvalues," IEEE Trans. Power Delivery, vol. 23, no. 4, pp. 2278-2285, Oct. 2008.

[44] T. Kailath, Linear Systems. Englewood Cliffs, NJ: Prentice Hall, 1980.

[45] C. T. Chen, Linear System Theory and Design. New York: Holt, Rinehart, Winston, 1984.

[46] J. M. Ortega and W. C. Rheinboldt, Iterative Solution of Nonlinear Equations in Several Variables. Boston: SIAM, 2000.

[47] W. A. Weiser and S. E. Zarantonello, "A note on piecewise linear and multilinear table interpolation in many dimensions," Mathematics of Computation, vol. 50, no. 181, pp. 253-264, Jan. 1988.

[48] D. F. Watson, "Computing the n-dimensional Delaunay tessellation with application to Voronoi polytopes," The Computer Journal, vol. 24, no. 2 , pp. 167-172, Febr. 1981.

[49] L. Pillegi, R. Rohrer, C. Visweswariah, Electronic Circuits and System Simulation Methods. McGraw-Hill Book Company, 1995.

[50] “Matlab User's Guide," The Mathworks, Inc., Natick, 2001.

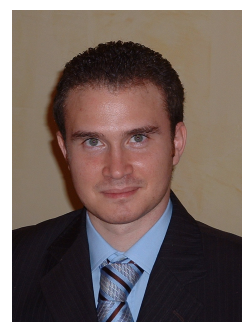

Francesco Ferranti (M'10) received the B.S. degree (summa cum laude) in electronic engineering from the Università degli Studi di Palermo, Palermo, Italy, in 2005, the M.S. degree (summa cum laude with honors) in electronic engineering from the Università degli Studi dell'Aquila, L'Aquila, Italy, in 2007, and the $\mathrm{Ph} . \mathrm{D}$. degree in electrical engineering from Ghent University, Ghent, Belgium, in 2011. He is currently an FWO Post-Doctoral Research Fellow with the Department of Information Technology (INTEC), Ghent University, Ghent, Belgium. His research interests include parametric macromodeling, parameterized model order reduction, signal integrity, electromagnetic compatibility, numerical modeling, and system identification.

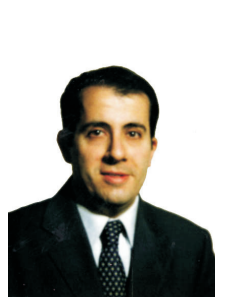

Giulio Antonini (M'94, SM'05) received his Laurea degree (summa cum laude) in Electrical Engineering in 1994 from the Università degli Studi dell'Aquila and the Ph.D. degree in Electrical Engineering in 1998 from University of Rome "La Sapienza". Since 1998 he has been with the UAq EMC Laboratory, Department of Electrical Engineering of the University of L'Aquila where he is currently Associate Professor. His research interests focus on EMC analysis, numerical modeling and in the field of signal integrity for high-speed digital systems. He has authored or co-authored more than 160 technical papers. Furthermore, he has given keynote lectures and chaired several special sessions at international conferences. He has been the recipient of the IEEE TRANSACTIONS ON ELECTROMAGNETIC COMPATIBILITY Best Paper Award in 1997, the CST University Publication Award in 2004, the IBM SHARED UNIVERSITY RESEARCH AWARD; in 2006 he has received a TECHNICAL ACHIEVEMENT AWARD from the IEEE EMC Society "for innovative contributions to computational electromagnetic on the Partial Element Equivalent Circuit (PEEC) technique for EMC applications". In 2008 he has received the IET-SMT best paper award. He holds one European Patent. Prof. Antonini is vice-chairman of the IEEE EMC Italy Chapter, he is member of the TC-9 committee and vice-chairman of the TC-10 Committee of the IEEE EMC Society. He serves as member of the Editorial Board of IET Science, Measurements and Technology. He serves as reviewer in a number of IEEE journals.

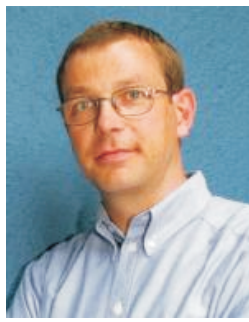

Tom Dhaene was born in Deinze, Belgium, on June 25,1966 . He received the Ph.D. degree in electrotechnical engineering from the University of Ghent, Ghent, Belgium, in 1993. From 1989 to 1993, he was Research Assistant at the University of Ghent, in the Department of Information Technology, where his research focused on different aspects of full-wave electro-magnetic circuit modeling, transient simulation, and time-domain characterization of high-frequency and high-speed interconnections. In 1993, he joined the EDA company Alphabit (now part of Agilent). He was one of the key developers of the planar EM simulator ADS Momentum. Since September 2000, he has been a Professor in the Department of Mathematics and Computer Science at the University of Antwerp, Antwerp, Belgium. Since October 2007, he is a Full Professor in the Department of Information Technology (INTEC) at Ghent University, Ghent, Belgium. As author or co-author, he has contributed to more than 150 peer-reviewed papers and abstracts in international conference proceedings, journals and books. He is the holder of 3 US patents. 


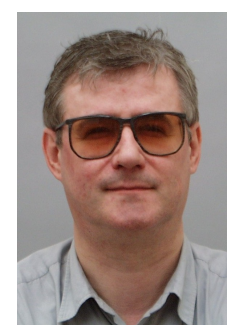

Luc Knockaert received the M. Sc. Degree in physical engineering, the M. Sc. Degree in telecommunications engineering and the Ph. D. Degree in electrical engineering from Ghent University, Belgium, in 1974, 1977 and 1987, respectively. From 1979 to 1984 and from 1988 to 1995 he was working in North-South cooperation and development projects at the Universities of the Democratic Republic of the Congo and Burundi. He is presently affiliated with the Interdisciplinary Institute for BroadBand Technologies (www.ibbt.be) and a professor at the Dept. of Information Technology, Ghent University (www.intec.ugent.be). His current interests are the application of linear algebra and adaptive methods in signal estimation, model order reduction and computational electromagnetics. As author or co-author he has contributed to more than 100 international journal and conference publications. He is a member of MAA, SIAM and a senior member of IEEE. 